\title{
Variational-Like Inequalities and Equilibrium Problems with Generalized Monotonicity in Banach Spaces
}

\author{
N. K. Mahato and C. Nahak \\ Department of Mathematics, Indian Institute of Technology Kharagpur, Kharagpur 721302, India \\ Correspondence should be addressed to C. Nahak, cnahak@maths.iitkgp.ernet.in
}

Received 22 September 2011; Accepted 16 November 2011

Academic Editor: Abdellah Bnouhachem

Copyright (C) 2012 N. K. Mahato and C. Nahak. This is an open access article distributed under the Creative Commons Attribution License, which permits unrestricted use, distribution, and reproduction in any medium, provided the original work is properly cited.

We introduce the notion of relaxed $(\rho-\theta)-\eta$-invariant pseudomonotone mappings, which is weaker than invariant pseudomonotone maps. Using the KKM technique, we establish the existence of solutions for variational-like inequality problems with relaxed $(\rho-\theta)-\eta$-invariant pseudomonotone mappings in reflexive Banach spaces. We also introduce the concept of $(\rho$ $\theta$ )-pseudomonotonicity for bifunctions, and we consider some examples to show that $(\rho-\theta)$ pseudomonotonicity generalizes both monotonicity and strong pseudomonotonicity. The existence of solution for equilibrium problem with $(\rho-\theta)$-pseudomonotone mappings in reflexive Banach spaces are demonstrated by using the KKM technique.

\section{Introduction}

Let $K$ be a nonempty subset of a real reflexive Banach space $X$, and let $X^{*}$ be the dual space of $X$. Consider the operator $T: K \rightarrow X^{*}$ and the bifunction $\eta: K \times K \rightarrow X$. Then the variational-like inequality problem (in short, VLIP) is to find $\bar{x} \in K$, such that

$$
\langle T \bar{x}, \eta(y, \bar{x})\rangle \geq 0, \quad \forall y \in K
$$

where $\langle\cdot, \cdot\rangle$ denote the pairing between $X$ and $X^{*}$.

If we take $\eta(x, y)=x-y$, then (1.1) becomes to find $\bar{x} \in K$, such that

$$
\langle T \bar{x}, y-\bar{x})\rangle \geq 0, \quad \forall y \in K
$$


which is classical variational inequality problems (VIPs). These problems have been studied in both finite and infinite dimensional spaces by many authors [1-3]. VIP has numerous applications in optimization, nonlinear analysis, and engineering sciences.

In the study of VLIP and VIP, monotonicity is the most common assumption for the operator $T$. Recently many authors established the existence of solutions for (VIP) and VLIP under generalized monotonicity assumptions, such as quasimonotonicity, relaxed monotonicity, densely pseudomonotonicity, relaxed $\eta$ - $\alpha$-monotonicity, and relaxed $\eta-\alpha$-pseudomonotonicity (see [1, 3-6] and the references therein). In 2008 [7], Behera et al. defined various concepts of generalized $(\rho-\theta)-\eta$-invariant monotonicities which are proper generalization of generalized invariant monotonicity introduced by Yang et al. [8]. Chen [9] defined semimonotonicity and studied semimonotone scalar variational inequalities problems in Banach spaces. Fang and Huang [3] obtained the existence of solution for VLIP using relaxed $\eta-\alpha$-monotone mappings in the reflexive Banach spaces. In [1], Bai et al. extended the results of [3] with relaxed $\eta$ - $\alpha$-pseudomonotone mappings and provided the existence of solution of the variational-like inequalities problems in reflexive Banach spaces. Bai et al. [10] studied variational inequalities problems with the setting of densely relaxed $\mu$ pseudomonotone operators and relaxed $\mu$-quasimonotone operators, respectively.

Inspired and motivated by $[1,3,10]$, we introduce the concept of relaxed $(\rho-\theta)$ $\eta$-invariant pseudomonotone mappings. Using the KKM technique, we establish the existence of solutions for Variational-like inequality problems with relaxed $(\rho-\theta)-\eta$-invariant pseudomonotone mappings. We also introduce the notion of $(\rho-\theta)$-pseudomonotonicity for bifunctions, and study some examples to show that $(\rho-\theta)$-pseudomonotonicity is proper generalization of monotonicity and the strong pseudomonotonicity. The existence of solutions of equilibrium problem with $(\rho-\theta)$-pseudomonotone mappings in reflexive Banach spaces are demonstrated, by using the KKM technique.

\section{Preliminaries}

Let $X$ be a real reflexive Banach space and $K$ be a nonempty subset of $X$, and $X^{*}$ be the space of all continuous linear functionals on $X$. Consider the functions $T: K \rightarrow X^{*}, \eta: K \times K \rightarrow X$ and $\theta: K \times K \rightarrow \mathbb{R}$ and $\rho \in \mathbb{R}$.

Definition 2.1. The operator $T: K \rightarrow X^{*}$ is said to be relaxed $(\rho-\theta)-\eta$-invariant pseudomonotone mapping with respect to $\eta$ and $\theta$, if for any pair of distinct points $x, y \in K$, one has

$$
\langle T x, \eta(y, x)\rangle \geq 0 \Longrightarrow\langle T y, \eta(x, y)\rangle+\rho|\theta(x, y)|^{2} \leq 0 \text {. }
$$

Remark 2.2. (i) If we take $\rho=0$ then from (2.1) it follows that $\langle T x, \eta(y, x)\rangle \geq 0 \Rightarrow$ $\langle T y, \eta(x, y)\rangle \leq 0$, for all $x, y \in K$, here $T$ is said to be invariant pseudomonotone, see [8].

(ii) If we take $\rho=0$, and $\eta(x, y)=x-y$, then (2.1) reduces to $\langle T x, y-x\rangle \geq 0 \Rightarrow$ $\langle T y, x-y\rangle \leq 0$, for all $x, y \in K$, and $T$ is said to be pseudomonotone map.

(iii) If $\theta(x, y)=\|x-y\|, \eta(x, y)=x-y, \rho<0$, that is, let $\rho=-\mu^{2}$ (where $\mu \in \mathbb{R}$ ).

Then (2.1) follows that $\langle T x, y-x)\rangle \geq 0 \Rightarrow\langle T y, y-x\rangle \geq-\mu^{2}\|x-y\|^{2}$, and $T$ is called relaxed $\mu$-pseudomonotone mapping [10].

It is obvious that every invariant pseudomonotone mapping is relaxed $(\rho-\theta)-\eta$ invariant pseudomonotone. However, the converse is not true in general, which is illustrated by the following counterexample. 
Example 2.3. Let $K=[0, \pi / 2]$ and $T:[0, \pi / 2] \rightarrow \mathbb{R}$ be defined by

$$
T x=\sin x-1
$$

Let the functions $\eta$ and $\theta$ be defined by

$$
\begin{gathered}
\eta(x, y)=\cos x-\cos y \\
\theta(y, x)= \begin{cases}\sqrt{\cos x-\cos y}, & x \leq y \\
1, & y<x\end{cases}
\end{gathered}
$$

Now, $\langle T x, \eta(y, x)\rangle=(\sin x-1)(\cos y-\cos x) \geq 0$, when $x \leq y$ or $x=\pi / 2$.

Take $\rho=-3$, then

$$
\begin{aligned}
\langle T y, \eta(x, y)\rangle-3|\theta(y, x)|^{2} & =(\sin y-1)(\cos x-\cos y)-3(\cos x-\cos y), \quad x \leq y \\
& =(\cos x-\cos y)(\sin y-4) \\
& \leq 0 .
\end{aligned}
$$

Therefore $T$ is relaxed $(\rho-\theta)-\eta$-invariant pseudomonotone mapping with respect to $\eta$ and $\theta$. But $T$ is not invariant pseudomonotone mapping with respect to the same $\eta$. In fact, if we take $x=\pi / 2$ and $y=0$. Therefore we have

$$
\langle T x, \eta(y, x)\rangle=0 .
$$

However,

$$
\langle T y, \eta(x, y)\rangle=(-1)\left(\cos \frac{\pi}{2}-\cos 0\right)=1>0
$$

Definition 2.4. The operator $T: K \rightarrow X^{*}$ is said to be relaxed $(\rho-\theta)$ - $\eta$-invariant quasimonotone mapping with respect to $\eta$ and $\theta$, if for any pair of distinct points $x, y \in K$, one has

$$
\langle T x, \eta(y, x)\rangle>0 \Longrightarrow\langle T y, \eta(x, y)\rangle+\rho|\theta(x, y)|^{2} \leq 0
$$

Next, we will show that relaxed $(\rho-\theta)-\eta$-invariant quasimonotonicity and relaxed $(\rho$ $\theta)-\eta$-invariant pseudomonotonicity coincide under some conditions. For this we need the following $\eta$-hemicontinuity definition. 
Definition 2.5 (see [3]). Let $T: K \rightarrow X^{*}$ and $\eta: K \times K \rightarrow X$. $T$ is said to be $\eta$-hemicontinuous if for any fixed $x, y \in K$, the mapping $f:[0,1] \rightarrow \mathbb{R}$ defined by $f(t)=\langle T(x+t(y-x)), \eta(y, x)\rangle$ is continuous at $0^{+}$.

Lemma 2.6. Let $T$ be an $\eta$-hemicontinuous and relaxed $(\rho-\theta)$ - $\eta$-invariant quasimonotone on $K$. Assume that the mapping $x \mapsto\langle T y, \eta(x, y)\rangle$ is concave and $\theta: K \times K \rightarrow \mathbb{R}$ is hemicontinuous in the second argument. Then for every $x, y \in K$ with $\langle T y, \eta(x, y)\rangle \geq 0$ one has either $\langle T x, \eta(y, x)\rangle+$ $\rho|\theta(y, x)|^{2} \leq 0$ or $\langle T y, \eta(z, y)\rangle \leq 0$, for all $z \in K$.

Proof. Suppose there exists some $z \in K$ such that $\langle T y, \eta(z, y)\rangle>0$. Then we have to prove that $\langle T x, \eta(y, x)\rangle+\rho|\theta(x, y)|^{2} \leq 0$.

Let $x_{t}=t z+(1-t) x, 0<t \leq 1$. Then

$$
\begin{aligned}
\left\langle T y, \eta\left(x_{t}, y\right)\right\rangle & \geq t\langle T y, \eta(z, y)\rangle+(1-t)\langle T y, \eta(x, y)\rangle \\
& >0 .
\end{aligned}
$$

Since $T$ is relaxed $(\rho-\theta)-\eta$-invariant quasimonotone on $K$, we have

$$
\left\langle T x_{t}, \eta\left(y, x_{t}\right)\right\rangle+\rho\left|\theta\left(y, x_{t}\right)\right|^{2} \leq 0
$$

Since $T$ is $\eta$-hemicontinuous and $\theta$ is hemicontinuous in the first variable and letting $t \rightarrow 0$, we have

$$
\langle T x, \eta(y, x)\rangle+\rho|\theta(y, x)|^{2} \leq 0
$$

Denote $K^{\perp}=\left\{z \in K^{*}:\langle z, \eta(x, y)\rangle=0\right.$, for all $\left.x, y \in K\right\}$, and

$$
T(K)=\{T x: x \in K\}
$$

Definition 2.7. A point $x_{0} \in K$ is said to be a $\eta$-positive point of $T: K \rightarrow X^{*}$ on $K$ if for all $x \in$ $K$, either $T x \in K^{\perp}$ or there exists $y \in K$ such that $\left\langle T x, \eta\left(y, x_{0}\right)\right\rangle>0$.

Let $K_{T}$ denotes the set of all $\eta$-positive points of $T$ on $K$. Now we give a characterization of relaxed $(\rho-\theta)-\eta$-invariant pseudomonotone.

Lemma 2.8. Let $T$ be $\eta$-hemicontinuous and relaxed $(\rho-\theta)$ - $\eta$-invariant quasimonotone on $K$ with $T(K) \cap K^{\perp}=\phi$. Assume that the mapping $x \mapsto\langle T y, \eta(x, y)\rangle$ is concave and $\theta$ is hemicontinuous. Then $T$ is relaxed $(\rho-\theta)$ - $\eta$-invariant pseudomonotone on $K_{T}$.

Proof. Let $x, y \in K_{T}$ with $\langle T x, \eta(y, x)\rangle \geq 0$. Therefore by the previous Lemma, we have either

$$
\langle T y, \eta(x, y)\rangle+\rho|\theta(x, y)|^{2} \leq 0 \quad \text { or } \quad\langle T x, \eta(z, y)\rangle \leq 0, \quad \forall z \in K
$$


Now we will show that the second inequality in (2.12) is impossible. In fact, since $x \in K_{T}$ and $T x \notin K^{\perp}$, there exists $z \in \mathrm{K}$ such that $\langle T x, \eta(z, x)\rangle>0$, which shows that the second inequality in (2.12) is impossible. Therefore,

$$
\langle T y, \eta(x, y)\rangle+\rho|\theta(x, y)|^{2} \leq 0
$$

hence $T$ is relaxed $(\rho-\theta)-\eta$-invariant pseudomonotone on $K_{T}$.

Remark 2.9. Lemmas 2.6 and 2.8 generalize Bai et al. [10] results [Lemma 2.1 and Proposition 2.1] from the case of relaxed $\mu$-quasimonotone operators to relaxed $(\rho-\theta)-\eta$ invariant quasimonotone operators.

Definition 2.10. Let $f: K \rightarrow 2^{X}$ be a set-valued mapping. Then $\mathrm{f}$ is said to be KKM mapping if for any $\left\{y_{1}, y_{2}, \ldots, y_{n}\right\}$ of $K$ one has $\operatorname{co}\left\{y_{1}, y_{2}, \ldots, y_{n}\right\} \subset \bigcup_{i=1}^{n} f\left(y_{i}\right)$, where $\operatorname{co}\left\{y_{1}, y_{2}, \ldots, y_{n}\right\}$ denotes the convex hull of $y_{1}, y_{2}, \ldots, y_{n}$.

Lemma 2.11 (see [11]). Let $M$ be a nonempty subset of a Hausdorff topological vector space $X$ and let $f: M \rightarrow 2^{X}$ be a KKM mapping. If $f(y)$ is closed in $X$ for all $y \in M$ and compact for some $y \in M$, then $\bigcap_{y \in M} f(y) \neq \phi$.

Theorem 2.12 (see [12]). Bounded, closed, convex subset of a reflexive Banach space is weakly compact.

\section{VLIP with Relaxed $(\rho-\theta)-\eta$-Invariant Pseudomonotonicity}

In this section, we establish the existence of the solution for VLIP, using relaxed $(\rho-\theta)-\eta$ invariant pseudomonotone mappings in reflexive Banach spaces.

Theorem 3.1. $T: K \rightarrow X^{*}$ is $\eta$-hemicontinuous and relaxed $(\rho-\theta)$ - $\eta$-invariant pseudomonotone mapping. Let the following hold:

(i) $\eta(x, y)+\eta(y, x)=0$, and $\theta(x, y)+\theta(y, x)=0$, for all $x, y \in K$;

(ii) $\theta(x, y)$ is convex in second argument and concave in first argument;

(iii) for a fixed $z, y \in K$, the mapping $x \mapsto\langle T z, \eta(x, y)\rangle$ is convex.

Then the following problems ( $a$ ) and (b) are equivalent:

(a) find $x \in K,\langle T x, \eta(y, x)\rangle \geq 0$, for all $y \in K$;

(b) find $x \in K,\langle T y, \eta(x, y)\rangle+\rho|\theta(x, y)|^{2} \leq 0$, for all $y \in K$.

Proof. Assume that $\bar{x}$ is a solution of (a). Therefore, (b) follows from the definition of relaxed $(\rho-\theta)-\eta$-invariant pseudomonotonicity of $T$.

Conversely, suppose that there exists an $\bar{x} \in K$ satisfying (b), that is,

$$
\langle T y, \eta(\bar{x}, y)\rangle+\rho|\theta(\bar{x}, y)|^{2} \leq 0, \quad \forall y \in K
$$

Choose any point $y \in K$ and consider $x_{t}=t y+(1-t) \bar{x}, t \in(0,1]$, then $x_{t} \in K$. 
Case I. When $\rho=0$.

Therefore from (3.1) we have

$$
\begin{gathered}
\left\langle T x_{t}, \eta\left(\bar{x}, x_{t}\right)\right\rangle \leq 0 \\
\left\langle T x_{t},-\eta\left(x_{t}, \bar{x}\right)\right\rangle \leq 0 \\
\left\langle T x_{t}, \eta\left(x_{t}, \bar{x}\right)\right\rangle \geq 0 .
\end{gathered}
$$

Now,

$$
\left\langle T x_{t}, \eta\left(x_{t}, \bar{x}\right)\right\rangle \leq t\left\langle T x_{t}, \eta(y, \bar{x})\right\rangle+(1-t)\left\langle T x_{t}, \eta(\bar{x}, \bar{x})\right\rangle=t\left\langle T x_{t}, \eta(y, \bar{x})\right\rangle
$$

From (3.2) and (3.3) we have

$$
\left\langle T x_{t}, \eta(y, \bar{x})\right\rangle \geq 0
$$

Since $T$ is $\eta$-hemicontinuous in the first argument and taking $t \rightarrow 0$ we get

$$
\langle T \bar{x}, \eta(y, \bar{x})\rangle \geq 0, \quad \forall y \in K
$$

Case II. When $\rho<0$, let $\rho=-k^{2}$.

From (3.1) we have

$$
\begin{aligned}
& \left\langle T x_{t}, \eta\left(\bar{x}, x_{t}\right)\right\rangle \leq k^{2}\left|\theta\left(\bar{x}, x_{t}\right)\right|^{2} \\
& \Longrightarrow\left\langle T x_{t}, \eta\left(x_{t}, \bar{x}\right)\right\rangle \geq-k^{2}\left|\theta\left(\bar{x}, x_{t}\right)\right|^{2} .
\end{aligned}
$$

From (ii), (iii), (3.3), and (3.6) we get,

$$
t\left\langle T x_{t}, \eta(y, \bar{x})\right\rangle \geq-k^{2} t^{2}|\theta(\bar{x}, y)|^{2} \text { which implies }\left\langle T x_{t}, \eta(y, \bar{x})\right\rangle \geq-k^{2} t|\theta(\bar{x}, y)|^{2}
$$

Since $T$ is $\eta$-hemicontinuous and taking $t \rightarrow 0$ we have

$$
\langle T \bar{x}, \eta(y, \bar{x})\rangle \geq 0, \quad \forall y \in K
$$

Case III. When $\rho>0$, let $\rho=k^{2}$.

From (3.1) we have

$$
\begin{aligned}
& \left\langle T x_{t}, \eta\left(\bar{x}, x_{t}\right)\right\rangle \leq-k^{2}\left|\theta\left(\bar{x}, x_{t}\right)\right|^{2} \\
& \Rightarrow\left\langle T x_{t}, \eta\left(x_{t}, \bar{x}\right)\right\rangle \geq k^{2}\left|\theta\left(\bar{x}, x_{t}\right)\right|^{2} .
\end{aligned}
$$


From (i), (ii), (iii),(3.3), and (3.9) we get,

$$
\left\langle T x_{t}, \eta(y, \bar{x})\right\rangle \geq k^{2} t|\theta(y, \bar{x})|^{2}
$$

Since $T$ is $\eta$-hemicontinuous and taking $t \rightarrow 0$ we have

$$
\langle T \bar{x}, \eta(y, \bar{x})\rangle \geq 0, \quad \forall y \in K
$$

Theorem 3.2. Let $K$ be a nonempty bounded closed convex subset of a real reflexive Banach space $X$. $T: K \rightarrow X^{*}$ is $\eta$-hemicontinuous and relaxed $(\rho-\theta)$ - $\eta$-invariant pseudomonotone mapping. Let the following hold:

(i) $\eta(x, y)+\eta(y, x)=0$, and $\theta(x, y)+\theta(y, x)=0$, for all $x, y \in K$;

(ii) $\theta(x, y)$ is convex in second argument and concave in first argument, and lower semicontinuous in the first argument;

(iii) for a fixed $z, y \in K$, the mapping $x \mapsto\langle T z, \eta(x, y)\rangle$ is convex and lower semicontinuous.

Then the problem (1.1) has a solution.

Proof. Consider the set valued mappings $F: K \rightarrow 2^{X}$ and $G: K \rightarrow 2^{X}$ such that

$$
\begin{gathered}
F(y)=\{x \in K:\langle T x, \eta(y, x)\rangle \geq 0\}, \quad \forall y \in K, \\
G(y)=\left\{x \in K:\langle T y, \eta(x, y)\rangle+\rho|\theta(x, y)|^{2} \leq 0\right\}, \quad \forall y \in K .
\end{gathered}
$$

It is easy to see that $\bar{x} \in K$ solves the VLIP if and only if $\bar{x} \in \bigcap_{y \in K} F(y)$. Thus it suffices to prove $\bigcap_{y \in K} F(y) \neq \phi$. To prove this, first we will show that $F$ is a KKM mapping.

If possible let $F$ not be a KKM mapping. Then there exists $\left\{x_{1}, x_{2}, \ldots, x_{m}\right\} \subset K$ such that $\operatorname{co}\left\{x_{1}, x_{2}, \ldots, x_{m}\right\} \notin \bigcup_{i=1}^{m} F\left(x_{i}\right)$, that means there exists a $x_{0} \in \operatorname{co}\left\{x_{1}, x_{2}, \ldots, x_{m}\right\}$, $x_{0}=\sum_{i=1}^{m} t_{i} x_{i}$ where $t_{i} \geq 0, i=1,2, \ldots, m, \sum_{i=1}^{m} t_{i}=1$, but $x_{0} \notin \bigcup_{i=1}^{m} F\left(x_{i}\right)$.

Hence, $\left\langle T x_{0}, \eta\left(x_{i}, x_{0}\right)\right\rangle<0$; for $i=1,2, \ldots, m$.

From (i) and (iii) it follows that

$$
0=\left\langle T x_{0}, \eta\left(x_{0}, x_{0}\right)\right\rangle \leq \sum_{i=1}^{m} t_{i}\left\langle T x_{0}, \eta\left(x_{i}, x_{0}\right)\right\rangle<0,
$$

which is a contradiction. Hence $F$ is a KKM mapping.

From the relaxed $(\rho-\theta)-\eta$-invariant pseudomonotonicity of $T$ it follows that $F(y) \subset$ $G(y)$, for all $y \in K$. Therefore $G$ is also a KKM mapping.

Since $K$ is closed bounded and convex, it is weakly compact. From the assumptions, we know that $G(y)$ is weakly closed for all $y \in K$. In fact, because $x \mapsto\langle T z, \eta(x, y)\rangle$ and $x \mapsto$ $\rho|\theta(x, y)|^{2}$ are lower semicontinuous. Therefore, $G(y)$ is weakly compact in $K$, for each $y \in$ $K$. Therefore from Lemma 2.11 and Theorem 3.1 it follows that $\bigcap_{y \in K} F(y)=\bigcap_{y \in K} G(y) \neq \phi$.So there exists $\bar{x} \in K$ such that $\langle T \bar{x}, \eta(y, \bar{x})\rangle \geq 0$, for all $y \in K$, that is, (1.1) has a solution. 
Theorem 3.3. Let $K$ be a nonempty unbounded closed convex subset of a real reflexive Banach space $X . T: K \rightarrow X^{*}$ is $\eta$-hemicontinuous and relaxed $(\rho-\theta)$ - $\eta$-invariant pseudomonotone mapping. Let the following hold:

(i) $\eta(x, y)+\eta(y, x)=0$, and $\theta(x, y)+\theta(y, x)=0$, for all $x, y \in K$;

(ii) $\theta(x, y)$ is convex in second argument and concave in first argument, and lower semicontinuous in the first argument;

(iii) for a fixed $z, y \in K$, the mapping $x \mapsto\langle T z, \eta(x, y)\rangle$ is convex and lower semicontinuous;

(iv) $T$ is weakly $\eta$-coercive, that is, there exits $x_{0} \in K$ such that $\left\langle T x, \eta\left(x, x_{0}\right)\right\rangle>0$, whenever $\|x\| \rightarrow \infty$ and $x \in K$.

Then (1.1) is solvable.

Proof. For $r>0$, assume $K_{r}=\{y \in K:\|y\| \leq r\}$.

Consider the problem: find $x_{r} \in K \cap K_{r}$ such that

$$
\left\langle T x_{r}, \eta\left(y, x_{r}\right)\right\rangle \geq 0, \quad \forall y \in K \cap K_{r} .
$$

By Theorem 3.2 we know that problem (3.14) has at least one solution $x_{r} \in K \cap K_{r}$. Choose $\left\|x_{0}\right\|<r$ with $x_{0}$ as in condition (iv). Then $x_{0} \in K \cap K_{r}$ and

$$
\left\langle T x_{r}, \eta\left(x_{0}, x_{r}\right)\right\rangle \geq 0
$$

From (i) we get,

$$
\left\langle T x_{r}, \eta\left(x_{0}, x_{r}\right)\right\rangle=-\left\langle T x_{r}, \eta\left(x_{r}, x_{0}\right)\right\rangle \text {. }
$$

If $\left\|x_{r}\right\|=r$ for all $r$, we may choose $r$ large enough so that by the assumption (iv) and (3.16) imply that $\left\langle T x_{r}, \eta\left(x_{0}, x_{r}\right)\right\rangle<0$, which contradicts (3.15).

Therefore there exists $r$ such that $\left\|x_{r}\right\|<r$. For any $y \in K$, we can choose $0<t<1$ small enough such that $x_{r}+t\left(y-x_{r}\right) \in K \cap K_{r}$.

From (3.14) it follows that

$$
\begin{aligned}
0 & \leq\left\langle T x_{r}, \eta\left(x_{r}+t\left(y-x_{r}\right), x_{r}\right)\right\rangle \\
& \leq t\left\langle T x_{r}, \eta\left(y, x_{r}\right)\right\rangle+(1-t)\left\langle T x_{r}, \eta\left(x_{r}, x_{r}\right)\right\rangle \\
& =t\left\langle T x_{r}, \eta\left(y, x_{r}\right)\right\rangle .
\end{aligned}
$$

Hence $\left\langle T x_{r}, \eta\left(y, x_{r}\right)\right\rangle \geq 0$, for all $y \in K$.

\section{Equilibrium Problem with $(\rho-\theta)$-Pseudomonotone Mappings}

Let $K$ be a nonempty subset of a real reflexive Banach space $X$, and consider the bifunction $f: K \times K \rightarrow \mathbb{R}$. Then the equilibrium problem (in short, $\mathrm{EP}$ ) is to find $\bar{x} \in K$, such that

$$
f(\bar{x}, y) \geq 0, \quad \forall y \in K
$$


Equilibrium problem was first introduced and studied by Blum and Oettli [2] in 1994. EP has many applications in nonlinear analysis, optimization, and game theory. The EP contains many problems as particular cases for examples, mathematical programming problems, complementary problems, Nash equilibrium problems in noncooperative games, variational inequality problems, fixed point problems, and minimax inequality problems.

Next we describe a number of particular cases of EP to explain our interest in EP, which have been discussed in [2].

(i) Optimization problem: let $\phi: K \rightarrow \mathbb{R}$, and consider minimization problem

(M) find $\bar{x} \in K$ such that $\phi(\bar{x}) \leq \phi(y)$, for all $y \in K$.

If we set $f(x, y)=\phi(y)-\phi(x)$, for all $x, y \in K$. Then problems EP and (M) are equivalent.

(ii) Variational inequality problem: if we define $f(x, y)=\langle T x, y-x\rangle$ where $T: K \rightarrow X^{*}$ is a given mapping, where $X^{*}$ denotes the space of all continuous linear maps on $X$. Then EP collapses into the classical VIP which states the following,

(VIP) find $\bar{x} \in X$ such that $\bar{x} \in K$, with $\langle T \bar{x}, y-\bar{x}\rangle \geq 0$, for all $y \in K$.

(iii) Fixed point problem: let $X$ be a Hilbert space, and $K$ is a nonempty closed convex subset of $X$. Let $T: K \rightarrow K$ be a given mapping. Then the fixed point problem is to

(FPP) find $\bar{x} \in K$ such that $\bar{x}=T \bar{x}$.

Set $f(x, y)=\langle x-T x, y-x\rangle$. Then $\bar{x}$ solves EP if and only if $\bar{x}$ is a solution of FPP.

The purpose of this section is to establish the existence of solution for equilibrium problems with $(\rho-\theta)$-pseudomonotone mappings in the reflexive Banach spaces. We first introduce the notion of $(\rho-\theta)$-monotone mappings and $(\rho-\theta)$-pseudomonotone mappings. We also provide some examples to justify that $(\rho-\theta)$-monotone mapping generalizes weakly monotone maps, and $(\rho-\theta)$-pseudomonotone mapping generalizes pseudomonotone, weakly pseudomonotone maps.

Let $K$ be a nonempty subset of a real reflexive Banach space $X$. Consider the function $f: K \times K \rightarrow \mathbb{R}$ and $\theta: K \times K \rightarrow \mathbb{R}$ and $\rho \in \mathbb{R}$.

Definition 4.1. The function $f: K \times K \rightarrow \mathbb{R}$ is said to be $(\rho-\theta)$-monotone with respect to $\theta: K \times K \rightarrow \mathbb{R}$ if, for all $x, y \in K$, one has

$$
f(x, y)+f(y, x) \leq \rho|\theta(x, y)|^{2}
$$

When

(i) $\rho>0$ and $\theta(x, y)=\|x-y\|, f$ is weakly monotone;

(ii) $\rho=0, f$ is monotone;

(iii) $\rho<0$ and $\theta(x, y)=\|x-y\|, f$ is strongly monotone.

We now give an example to show that $(\rho-\theta)$ monotonicity is a generalization of both monotonicity and weakly monotonicity. 
Example 4.2. Let $K=[1,10]$. Let the functions $f$ and $\theta$ be defined by

$$
f(x, y)=x^{2}+y^{2}+1, \quad \theta(x, y)=\sqrt{2\left(x^{2}+y^{2}\right)+4} .
$$

Then

$$
\begin{aligned}
f(x, y)+f(y, x) & =2\left(x^{2}+y^{2}+1\right) \\
& \leq \rho\left(2 x^{2}+2 y^{2}+4\right), \quad \text { for any } \rho \geq 1
\end{aligned}
$$

Therefore $f$ is $(\rho-\theta)$-monotone with respect to $\theta$.

There exists no constant $\rho>0$ such that $f(x, y)+f(y, x) \leq \rho\|x-y\|^{2}$, for all $x, y \in K$. As if we assume $x$ and $y$ to be such that their difference is very small, then right-hand side of the inequality tends to zero and left-hand side is always greater than 2 . Hence $f(x, y)$ is not weakly monotone. Again since $f$ is positive valued, $f$ is not monotone.

Definition 4.3. The function $f: K \times K \rightarrow \mathbb{R}$ is said to be $(\rho-\theta)$-pseudomonotone with respect to $\theta$ if for any pair of distinct points $x, y \in K$, one has

$$
f(x, y) \geq 0 \text { implies } f(y, x) \leq \rho|\theta(x, y)|^{2} .
$$

Every $(\rho-\theta)$-monotone mapping is a $(\rho-\theta)$-pseudomonotone with respect to the same $\rho$ and $\theta$. However, the converse is not true in general, which follows from the following counterexample.

Example 4.4. Let the functions $f:(0, \pi / 2) \times(0, \pi / 2) \rightarrow \mathbb{R}$ and $\theta:(0, \pi / 2) \times(0, \pi / 2) \rightarrow \mathbb{R}$ be defined by

$$
f(x, y)=(\cos y+6)^{2}-\cos ^{2} x, \quad \theta(x, y)=\sqrt{50+\cos x-\cos y} \text {, respectively. }
$$

Take $\rho=1$. We have to show

$$
\begin{gathered}
f(x, y) \geq 0 \quad \text { implies } f(y, x) \leq \rho|\theta(x, y)|^{2} . \\
f(x, y)=(\cos y+6)^{2}-\cos ^{2} x \geq 0, \quad \forall x, y \in\left(0, \frac{\pi}{2}\right) .
\end{gathered}
$$

Now,

$$
\begin{aligned}
f(y, x)-\rho|\theta(x, y)|^{2} & =(\cos x+6)^{2}-\cos ^{2} y-(50+\cos x-\cos y) \\
& =(\cos x+6)^{2}+\cos y-\left(50+\cos ^{2} y+\cos x\right) \\
& \leq 0
\end{aligned}
$$


Hence $f$ is $(\rho-\theta)$-pseudomonotone mapping with respect to $\theta$. But $f$ is not $(\rho-\theta)$-monotone mapping with respect to the same $\theta$. In fact,

$$
\begin{aligned}
f(x, y)+f(y, x) & =(\cos y+6)^{2}+(\cos x+6)^{2}-\left(\cos ^{2} x+\cos ^{2} y\right) \\
& \not|\theta(x, y)|^{2}=(50+\cos x-\cos y) .
\end{aligned}
$$

Note that in the above example, $f$ is neither a monotone nor pseudomonotone mapping.

Definition 4.5. The function $f: K \times K \rightarrow \mathbb{R}$ is said to be $(\rho-\theta)$-quasimonotone with respect to $\theta$ if for any pair of distinct points $x, y \in K$, one has

$$
f(x, y)>0 \text { implies } f(y, x) \leq \rho|\theta(x, y)|^{2} .
$$

Next, we will show that $(\rho-\theta)$-quasimonotonicity and $(\rho-\theta)$-pseudomonotonicity are equivalent under certain conditions.

Lemma 4.6. Let $f$ be hemicontinuous and $(\rho-\theta)$-quasimonotone on $K$. Assume that $f$ is concave in the second argument and $\theta$ is hemicontinuous in the second argument. Then for every $x, y \in K$ with $f(y, x) \geq 0$ one has either $f(x, y) \leq \rho|\theta(y, x)|^{2}$ or $f(y, z) \leq 0$, for all $z \in K$.

Proof. Suppose there exists some $z \in K$ such that $f(y, z)>0$. Then we have to prove that $f(x, y) \leq \rho|\theta(x, y)|^{2}$.

$$
\text { Let } x_{t}=t z+(1-t) x, 0<t \leq 1 \text {. Then }
$$

$$
\begin{aligned}
f\left(y, x_{t}\right) & \geq t f(y, z)+(1-t) f(y, x) \\
& >0 .
\end{aligned}
$$

Since $T$ is relaxed $(\rho-\theta)$-quasimonotone on $K$, it implies that

$$
f\left(x_{t}, y\right) \leq \rho\left|\theta\left(y, x_{t}\right)\right|^{2}
$$

Now letting $t \rightarrow 0$, we have

$$
f(x, y) \leq \rho|\theta(y, x)|^{2}
$$

This completes the proof.

Theorem 4.7. Let $K$ be a nonempty convex subset of a real reflexive Banach space $X$. Suppose $f$ : $K \times K \rightarrow \mathbb{R}$ is $(\rho-\theta)$-pseudomonotone with respect to $\theta$ and is hemicontinuous in the first argument with the following conditions:

(i) $f(x, x)=0$, for all $x \in K$;

(ii) for fixed $z \in K$, the mapping $x \mapsto f(z, x)$ is convex; 
(iii) $\theta(x, y)+\theta(y, x)=0$, for all $x, y \in K$;

(iv) $\theta$ is convex in first argument and concave in the second argument.

Then $\bar{x} \in K$ is a solution of (4.1) if and only if

$$
f(y, \bar{x}) \leq \rho|\theta(\bar{x}, y)|^{2}, \quad \forall y \in K
$$

Proof. Assume that $\bar{x}$ is a solution of (4.1) that is, $f(\bar{x}, y) \geq 0$, for all $y \in K$. Therefore from the definition of $(\rho-\theta)$ pseudomonotonicity of $T$ it follows that

$$
f(y, \bar{x}) \leq \rho|\theta(\bar{x}, y)|^{2}, \quad \forall y \in K
$$

Conversely, suppose $\exists \bar{x} \in K$ satisfying (4.14), that is,

$$
f(y, \bar{x}) \leq \rho|\theta(\bar{x}, y)|^{2}, \quad \forall y \in K \text {. }
$$

Choose any point $y \in K$ and consider $x_{t}=t y+(1-t) \bar{x}, t \in(0,1]$, then $x_{t} \in K$.

Case I. When $\rho=0$.

Therefore from (4.16) we have

$$
f\left(x_{t}, \bar{x}\right) \leq 0, \quad \forall y \in K
$$

Now conditions (i) and (ii) imply that,

$$
\begin{aligned}
0 & =f\left(x_{t}, x_{t}\right) \leq t f\left(x_{t}, y\right)+(1-t) f\left(x_{t}, \bar{x}\right) \\
& \Longrightarrow t\left[f\left(x_{t}, \bar{x}\right)-f\left(x_{t}, y\right)\right] \leq f\left(x_{t}, \bar{x}\right) .
\end{aligned}
$$

From (4.17) and (4.18) we have

$$
f\left(x_{t}, \bar{x}\right)-f\left(x_{t}, y\right) \leq 0, \quad \forall y \in K
$$

Since $f$ is hemicontinuous in the first argument and taking $t \rightarrow 0$, it implies that

$$
f(\bar{x}, y) \geq 0, \quad \forall y \in K
$$

Hence $\bar{x}$ is a solution of (4.1).

Case II. When $\rho<0$, let $\rho=-k^{2}$.

From (4.16) we have

$$
f\left(x_{t}, \bar{x}\right) \leq-k^{2}\left|\theta\left(\bar{x}, x_{t}\right)\right|^{2} .
$$


Now using (4.18), (4.21), and (iv) it follows that

$$
f\left(x_{t}, \bar{x}\right)-f\left(x_{t}, y\right) \leq-k^{2} t|\theta(\bar{x}, y)|^{2} .
$$

Since $f$ is hemicontinuous in the first argument and letting $t \rightarrow 0$, we get

$$
f(\bar{x}, y) \geq 0, \quad \forall y \in K
$$

Case III. When $\rho>0$, let $\rho=k^{2}$.

From (4.16), (4.18), and (iv) we have

$$
\begin{gathered}
f\left(x_{t}, \bar{x}\right) \leq k^{2}\left|\theta\left(\bar{x}, x_{t}\right)\right|^{2}, \\
\Rightarrow f\left(x_{t}, \bar{x}\right)-f\left(x_{t}, y\right) \leq k^{2} t|\theta(y, \bar{x})|^{2} .
\end{gathered}
$$

Since $f$ is hemicontinuous in the first argument and taking $t \rightarrow 0$, we get

$$
f(\bar{x}, y) \geq 0, \quad \forall y \in K
$$

Theorem 4.8. Let $K$ be a nonempty bounded convex subset of a real reflexive Banach space $X$. Suppose $f: K \times K \rightarrow \mathbb{R}$ is $(\rho-\theta)$-pseudomonotone with respect to $\theta$ and is hemicontinuous in the first argument with the following conditions:

(i) $f(x, x)=0$, for all $x \in K$;

(ii) for fixed $z \in K$, the mapping $x \mapsto f(z, x)$ is convex and lower semicontinuous;

(iii) $\theta(x, y)+\theta(y, x)=0$, for all $x, y \in K$;

(iv) $\theta$ is convex in first argument and concave in the second argument, and lower semicontinuous in the first argument.

Then the problem (4.1) has a solution.

Proof. Consider the two set valued mappings $F: K \rightarrow 2^{X}$ and $G: K \rightarrow 2^{X}$ such that

$$
\begin{gathered}
F(y)=\{x \in K: f(x, y) \geq 0\}, \quad \forall y \in K, \\
G(y)=\left\{x \in K: f(y, x) \leq \rho|\theta(x, y)|^{2}\right\}, \quad \forall y \in K .
\end{gathered}
$$

It is easy to see that $\bar{x} \in K$ solves the equilibrium problem (4.1) if and only if $\bar{x} \in \bigcap_{y \in K} F(y)$. First to show that $F$ is a KKM mapping. If possible let $F$ not be a KKM mapping. Then there exists $\left\{x_{1}, x_{2}, \ldots, x_{m}\right\} \subset K$ such that $\operatorname{co}\left\{x_{1}, x_{2}, \ldots, x_{m}\right\} \nsubseteq \bigcup_{i=1}^{m} F\left(x_{i}\right)$, that means there exists a $x_{0} \in \operatorname{co}\left\{x_{1}, x_{2}, \ldots, x_{m}\right\}, x_{0}=\sum_{i=1}^{m} t_{i} x_{i}$ where $t_{i} \geq 0, i=1,2, \ldots, m$, $\sum_{i=1}^{m} t_{i}=1$, but $x_{0} \notin \bigcup_{i=1}^{m} F\left(x_{i}\right)$. Hence, $f\left(x_{0}, x_{i}\right)<0$; for $i=1,2, \ldots, m$. 
From (i) and (ii) it follows that

$$
0=f\left(x_{0}, x_{0}\right) \leq \sum_{i=1}^{m} t_{i} f\left(x_{0}, x_{i}\right)<0,
$$

which is a contradiction. Hence $F$ is a KKM mapping.

From the $(\rho-\theta)$-pseudomonotonicity of $f$ it follows that $F(y) \subset G(y)$, for all $y \in K$. Therefore $G$ is also a KKM mapping.

Since $K$ is closed bounded and convex, it is weakly compact. From the assumptions, we know that $G(y)$ is weakly closed for all $y \in K$. In fact, because $x \mapsto f(z, x)$ and $x \mapsto \rho \mid\left(\left.\theta(x, z)\right|^{2}\right.$ are lower semicontinuous. Therefore, $G(y)$ is weakly compact in $K$, for each $y \in K$

Therefore from Lemma 2.11 and Theorem 4.7 it follows that $\bigcap_{y \in K} F(y)=$ $\bigcap_{y \in K} G(y) \neq \phi$.

So there exists $\bar{x} \in K$ such that $f(\bar{x}, y) \geq 0$, for all $y \in K$, that is, (4.1) has a solution.

Theorem 4.9. Let $K$ be a nonempty unbounded closed convex subset of a real reflexive Banach space $X$. Suppose $f: K \times K \rightarrow \mathbb{R}$ is $(\rho-\theta)$-pseudomonotone with respect to $\theta$ and is hemicontinuous in the first argument and satisfy the following assumptions:

(i) $f(x, x)=0$, for all $x \in K$;

(ii) for fixed $z \in K$, the mapping $x \mapsto f(z, x)$ is convex and lower semicontinuous;

(iii) $\theta(x, y)+\theta(y, x)=0$, for all $x, y \in K$;

(iv) $\theta$ is convex in first argument and concave in the second argument, and lower semicontinuous in the first argument.

(v) $f$ is weakly coercive, that is, there exists $x_{0} \in K$ such that

$$
f\left(x, x_{0}\right)<0, \quad \text { whenever }\|x\| \rightarrow+\infty \text {, an } x \in K \text {. }
$$

Then (4.1) has a solution.

Proof. For $r>0$, assume $K_{r}=\{y \in K:\|y\| \leq r\}$.

Consider the problem: find $x_{r} \in K \cap K_{r}$ such that

$$
f\left(x_{r}, y\right) \geq 0, \quad \forall y \in K \cap K_{r}
$$

By Theorem 4.8 we know that the problem (4.29) has at least one solution $x_{r} \in K \cap K_{r}$.

Choose $\left\|x_{0}\right\|<r$ with $x_{0}$ as in condition (v). Then $x_{0} \in K \cap K_{r}$ and

$$
f\left(x_{r}, x_{0}\right) \geq 0 \text {. }
$$

If $\left\|x_{r}\right\|=r$ for all $r$, we may choose $r$ large enough so that by the assumption (v) imply that $f\left(x_{r}, x_{0}\right)<0$, which contradicts (4.30). 
Therefore there exists $r$ such that $\left\|x_{r}\right\|<r$. For any $y \in K$, we can choose $0<t<1$ small enough such that $x_{r}+t\left(y-x_{r}\right) \in K \cap K_{r}$.

From (4.29) it follows that

$$
\begin{aligned}
0 & \leq f\left(x_{r}, x_{r}+t\left(y-x_{r}\right)\right) \\
& \leq t f\left(x_{r}, y\right)+(1-t) f\left(x_{r}, x_{r}\right) \\
& =t f\left(x_{r}, y\right)
\end{aligned}
$$

Hence $f\left(x_{r}, y\right) \geq 0$, for all $y \in K$.

\section{Conclusions}

The present work has been aimed to theoretically study the existence of solutions for variational-like inequality problems under a new concept relaxed $(\rho-\theta)-\eta$-invariant pseudomonotone maps in reflexive Banach spaces. We have also obtained existence of solutions of equilibrium problems with $(\rho-\theta)$-pseudomonotone mappings. More research and development activities is therefore needed on generalized monotonicity to demonstrate the equilibrium problem and variational inequality problem.

\section{Acknowledgments}

The authors are very much thankful to the editor and referees for their suggestions which helped to improve the presentation of this paper. The work of the authors was partially supported by CSIR, New Delhi, Grant 25 (0163)/08/EMR-II.

\section{References}

[1] M.-R. Bai, S.-Z. Zhou, and G.-Y. Ni, "Variational-like inequalities with relaxed $\eta$ - $\alpha$ pseudomonotone mappings in Banach spaces," Applied Mathematics Letters, vol. 19, no. 6, pp. 547-554, 2006.

[2] E. Blum and W. Oettli, "From optimization and variational inequalities to equilibrium problems," The Mathematics Student, vol. 63, no. 1-4, pp. 123-145, 1994.

[3] Y. P. Fang and N. J. Huang, "Variational-like inequalities with generalized monotone mappings in Banach spaces," Journal of Optimization Theory and Applications, vol. 118, no. 2, pp. 327-338, 2003.

[4] B.-S. Lee and G.-M. Lee, "Variational inequalities for $(\eta, \theta)$-pseudomonotone operators in nonreflexive Banach spaces," Applied Mathematics Letters, vol. 12, no. 5, pp. 13-17, 1999.

[5] D. T. Luc, "Existence results for densely pseudomonotone variational inequalities," Journal of Mathematical Analysis and Applications, vol. 254, no. 1, pp. 291-308, 2001.

[6] N. Hadjisavvas and S. Schaible, "Quasimonotone variational inequalities in Banach spaces," Journal of Optimization Theory and Applications, vol. 90, no. 1, pp. 95-111, 1996.

[7] N. Behera, C. Nahak, and S. Nanda, "Generalized $(\rho-\theta)-\eta$-invexity and generalized $(\rho-\theta)-\eta$-invariantmonotonicity," Nonlinear Analysis: Theory, Methods E Applications, vol. 68, no. 8, pp. 2495-2506, 2008.

[8] X. M. Yang, X. Q. Yang, and K. L. Teo, "Generalized invexity and generalized invariant monotonicity," Journal of Optimization Theory and Applications, vol. 117, no. 3, pp. 607-625, 2003.

[9] Y.-Q. Chen, "On the semi-monotone operator theory and applications," Journal of Mathematical Analysis and Applications, vol. 231, no. 1, pp. 177-192, 1999.

[10] M.-R. Bai, S.-Z. Zhou, and G.-Y. Ni, "On the generalized monotonicity of variational inequalities," Computers \& Mathematics with Applications, vol. 53, no. 6, pp. 910-917, 2007.

[11] K. Fan, "Some properties of convex sets related to fixed point theorems," Mathematische Annalen, vol. 266, no. 4, pp. 519-537, 1984.

[12] H. Brezis, Analyse Fonctionnelle: Théorie et Applications, Collection Mathématiques Appliquées pour la Maîtrise, Masson, Paris, France, 1983. 


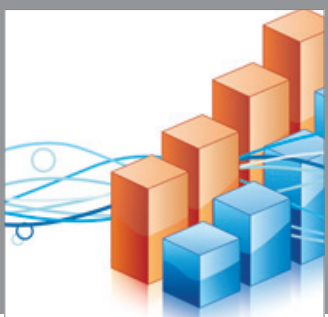

Advances in

Operations Research

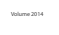

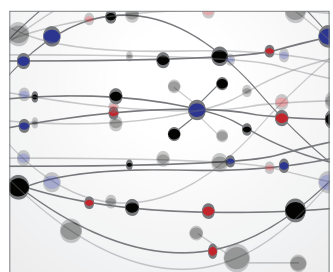

\section{The Scientific} World Journal
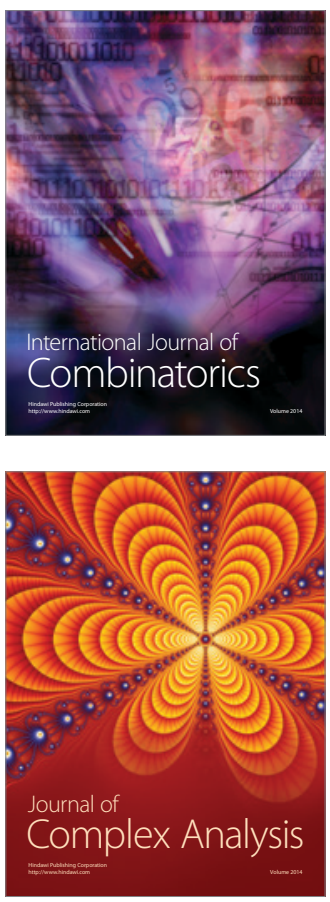

International Journal of

Mathematics and

Mathematical

Sciences
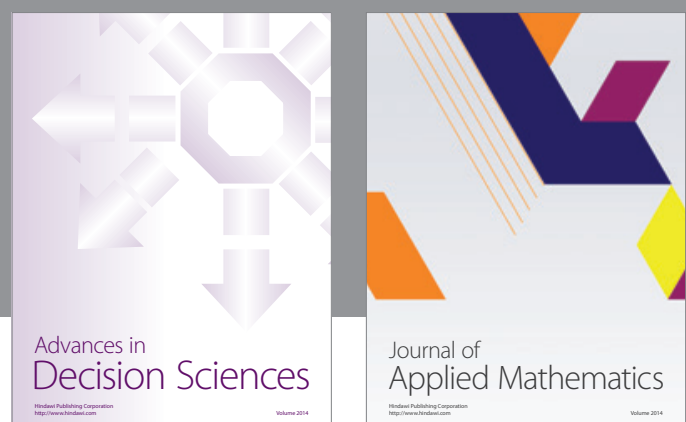

Journal of

Applied Mathematics
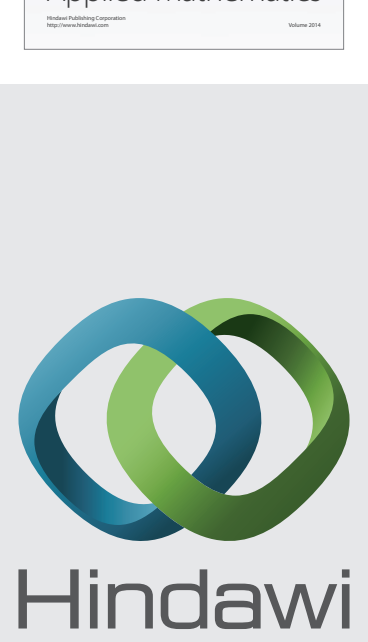

Submit your manuscripts at http://www.hindawi.com
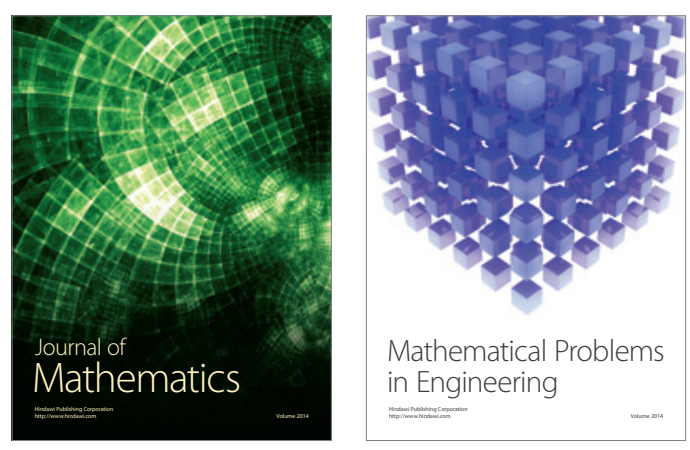

Mathematical Problems in Engineering
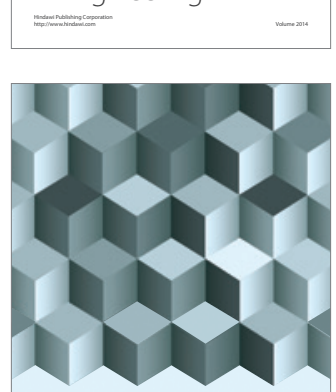

Journal of

Function Spaces
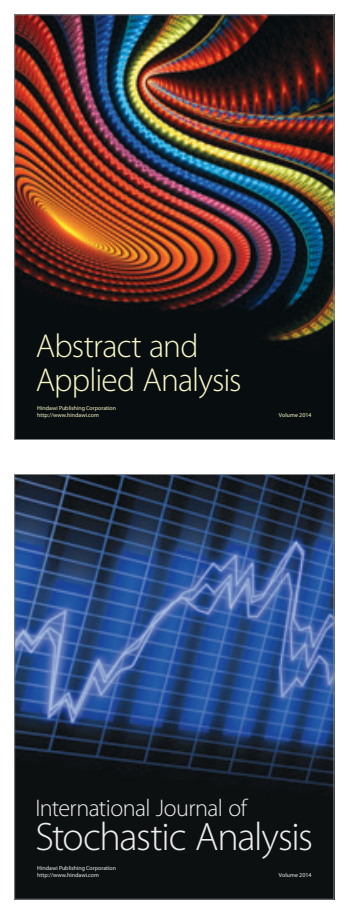

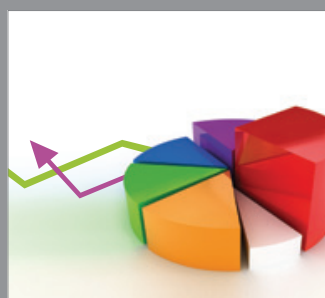

ournal of

Probability and Statistics

Promensencen
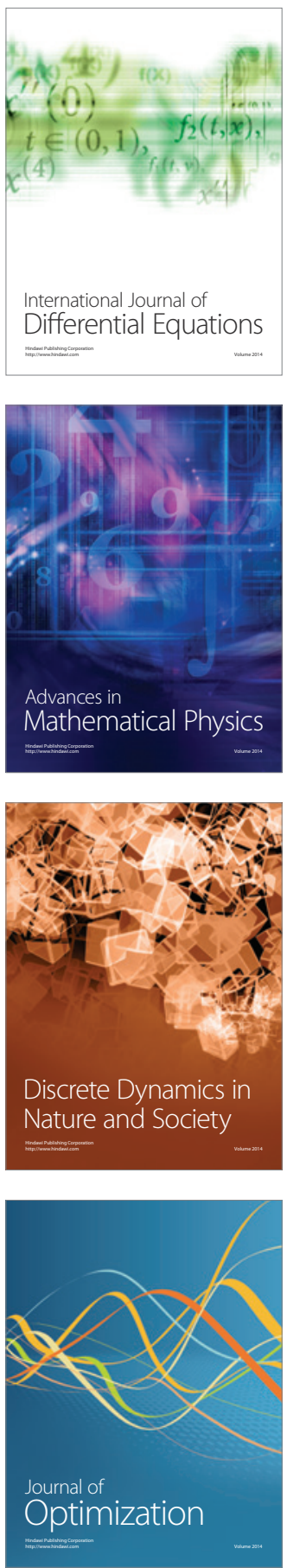